\title{
Influencia de la concentración sobre las temperaturas de la transformación martensítica en las aleaciones de base cobre con memoria de forma
}

\author{
V. Recarte ${ }^{(*)}$, R.B. Pérez-Sáez ${ }^{(*)}$, E. Hernández-Bocanegra ${ }^{(* *)}$, M.L. Nó ${ }^{(* *)}$ y J. San Juan ${ }^{(*)}$
}

Resumen La composición de la aleación es el principal factor determinante de las temperaturas características de la transformación martensítica, siendo éstas muy sensibles a las variaciones de concentración de los elementos aleantes. Este hecho es muy importante desde el punto de vista tecnológico, dada la dificultad para fijar la composición de una aleación ternaria o cuaternaria con gran precisión. Por ello, se ha establecido una relación empírica entre la concentración de los elementos aleantes y las temperaturas de transformación martensítica aplicable dentro del rango de composiciones de interés tecnológico en las aleaciones con memoria de forma de $\mathrm{Cu}-\mathrm{Al}-\mathrm{Ni}$.

Palabras clave: Transformación martensítica. Memoria de forma. Cu-Al-Ni. Fases martensíticas.

\section{Influence of concentration on the martensitic transformation temperatures in $\mathrm{Cu}$-based shape memory alloys}

\begin{abstract}
The alloy composition is the most important factor that determines the characteristic martensitic transformation temperatures in shape memory alloys. These temperatures are very sensitive to a change of alloying element concentrations. From a technological point of view this fact is very important due to the difficulty to control with high precision the concentration in ternary or quaternary alloys. An empirical relationship between the alloying element concentrations and the martensitic transformation temperatures has been stablished for $\mathrm{Cu}-\mathrm{Al}-\mathrm{Ni}$ shape memory alloys.
\end{abstract}

Keywords: Martensitic transformation. Shape memory. Cu-Al-Ni. Martensite phases.

\section{INTRODUCCIÓN}

La utilización de las aleaciones con memoria de forma en aplicaciones comerciales requiere la reproducibilidad de la transformación martensítica responsable de las propiedades termomecánicas de estas aleaciones, si se quieren cumplir los requisitos de fiabilidad impuestos por las aplicaciones tecnológicas. Ello conlleva, en particular, el control preciso de las temperaturas de transformación y en especial de $M_{\mathrm{s}}$.

(*) Dpto. Física Mat. Condensada. Fac. Ciencias. Univ. País Vasco. Aptdo. 644. 48080-Bilbao (España).

(**) Dpto. Física Aplicada II. Fac. Ciencias. Univ. País Vasco. Aptdo. 644. 48080-Bilbao (España).
La composición de la aleación es el principal factor determinante de la temperatura $M_{\mathrm{s}}$, siendo ésta muy sensible a las variaciones de concentración de los elementos aleantes. Esta característica es muy importante desde el punto de vista tecnológico, dada la dificultad en fijar la composición de una aleación ternaria o cuaternaria con gran precisión. La dependencia de $M_{\mathrm{s}}$ con la composición está ligada al cambio de la diferencia entre las energías libres de la martensita y la fase $\beta$ con la misma. En dicha diferencia, aparecen factores de tipo químico, electrónico y estructurales. Por ello, a pesar de que en las aleaciones con memoria de forma de base cobre, la estabilidad relativa de las fases de equilibrio $\alpha, \beta, \gamma$, está controlada principalmente por la concentración electrónica e/a, no se ha encontrado 
relación entre e/a y $M_{\mathrm{s}}$ (1 y 2). Por otra parte, Ahlers (2) establece una relación entre $M$ s y la distorsión ortorrómbica medida a través del ángulo $\beta$ de la martensita en aleaciones binarias y ternarias basadas en el $\mathrm{Cu}-\mathrm{Zn}$.

En las aleaciones de $\mathrm{Cu}-\mathrm{Al}-\mathrm{Ni}$ hay pocos trabajos dedicados al estudio de la influencia de la composición en las temperaturas de transformación (3 y 4). De hecho, no existe ninguna referencia que cite una ecuación empírica del tipo de las expuestas anteriormente que relacione $M_{\mathrm{s}}$ con el porcentaje de níquel y aluminio. Por lo tanto, presenta gran interés el establecemiento de la evolución de las temperaturas de transformación, en función de la composición, sobre muestras sometidas al mismo tratamiento termomecánico y a las mismas condiciones de medida.

\section{TÉCNICAS EXPERIMENTALES}

El trabajo se ha realizado sobre las aleaciones monocristalinas cuyas concentraciones, en $\%$ en masa, se pueden agrupar en tres series. La serie 1 , en la que se mantiene el níquel constante al $4 \%$, variando el aluminio del 13,2 al 14,2\%. La serie 2 , en la que se mantiene el aluminio constante al 13,7 $\%$, variando el níquel del 3 al $5 \%$ y la serie 3 , en la que se mantiene fijo en el $13,2 \%$ el contenido de aluminio y se varía el de níquel del 3,5 al 5,5\%. De esta forma, las tres series presentan concentraciones cruzadas, que abarcan una parte importante del rango de concentraciones de interés tecnológico (5).

Las medidas de calorimetría diferencial de barrido (D.S.C.) se han realizado en un calorímetro Perkin Elmer DSC7 a una velocidad de calentamiento de $10^{\circ} \mathrm{C} / \mathrm{min}$. Con el fin de poder comparar los resultados, todas las muestras han sido sometidas al mismo tratamiento térmico: $20 \mathrm{~min}$ a $900{ }^{\circ} \mathrm{C}+$ temple en $\mathrm{H}_{2} \mathrm{O}$ a $0{ }^{\circ} \mathrm{C}$. Con el fin de determinar las temperaturas de transformación, se han tomado el 2 y el $98 \%$ de la fracción volumétrica transformada obtenida a partir de la integral de la entropía DS normalizada (6).

\section{RESULTADOS EXPERIMENTALES}

Se han observado tres tipos de comportamiento de la transformación:

- Una transformación que se produce de forma suave y continua, con una histéresis muy baja, $\approx$ $10{ }^{\circ} \mathrm{C}$. Su curva de fracción volumétrica presenta un comienzo y un final suaves, con un intervalo de temperatura de transformación de aproximadamente $40{ }^{\circ} \mathrm{C}$ (Fig. 1c). Este comportamiento es propio de una transformación $\beta_{3} \Leftrightarrow \beta_{3}$.
- Una transformación abrupta que da lugar a varios picos agudos, presentando un carácter menos suave y progresivo que la anterior y con una histéresis de 30 ó $40^{\circ} \mathrm{C}$ (Fig. 1 a). La fracción volumétrica asociada presenta un comienzo y un final abruptos, con un intervalo de la temperatura de transformación de $10^{\circ} \mathrm{C}$. Igualmente presenta escalones que indican un proceso de transformación en forma de avalanchas. Este comportamiento es propio de una transformación $\beta_{3} \Leftrightarrow \gamma_{3}$.

- Una transformación donde aparecen ambas características y que presenta dos picos en la transformación inversa (Fig. 1 b). Esto apuntaría a que coexisten ambas transformaciones. Este comportamiento es propio de una transformación mixta $\beta_{3} \Leftrightarrow \beta_{3}{ }^{\prime}+\gamma_{3}$.

En la figura 2 se muestra la dependencia de $M_{\mathrm{s}} \mathrm{y}$ $M_{\mathrm{f}}$ con la concentración de aluminio para las aleaciones de la serie 1 (Fig. $2 a$ ) y la dependencia con respecto a la concentración de níquel para la serie 2 (Fig. 2 b). Un incremento en la concentración de ambos elementos aleantes disminuye las temperaturas de transformación. Igualmente, se observa una dependencia mucho mayor de $M_{\mathrm{s}}$ con la concentración de aluminio que con la de níquel. En la serie 1, para las aleaciones con menor concentración de aluminio, el intervalo de transformación, $M \mathrm{~s}-M_{\mathrm{f}}$, aumenta, debido a que se pasa de una transformación $\beta_{3} \Leftrightarrow \gamma^{6}$, para las muestras ricas en aluminio, a una transformación $\beta_{3} \Leftrightarrow \beta^{\prime}{ }_{3}$ para las aleaciones pobres en dicho elemento, siendo mayor el intervalo de este último tipo de transformación (Fig. 1 c). Además, existe un rango intermedio de concentraciones donde se produce la transformación mixta $\beta_{3} \Leftrightarrow \beta_{3}{ }_{3}+\gamma^{6}{ }_{3}$.

\section{RELACIÓN EMPÍRICA DE $M_{S}$ CON LA CONCENTRACIÓN DE NÍQUEL Y DE ALUMINIO}

Mediante regresión lineal múltiple se ha obtenido la siguiente relación entre las temperaturas de transformación medidas y $M_{\mathrm{s}}$ :

$$
M_{\mathrm{S}}=2.433-169,6 \times \% \mathrm{Al}-19,1 \times \% \mathrm{Ni} \text { (en masa) }
$$

o bien tomando porcentajes atómicos:

$M_{\mathrm{S}}=2.871-101,5 \times \% \mathrm{Al}-22,9 \times \% \mathrm{Ni}$ (atómico)

Con estos ajustes, las temperaturas medidas difieren en menos de $\pm 8{ }^{\circ} \mathrm{C}$ de los valores de 

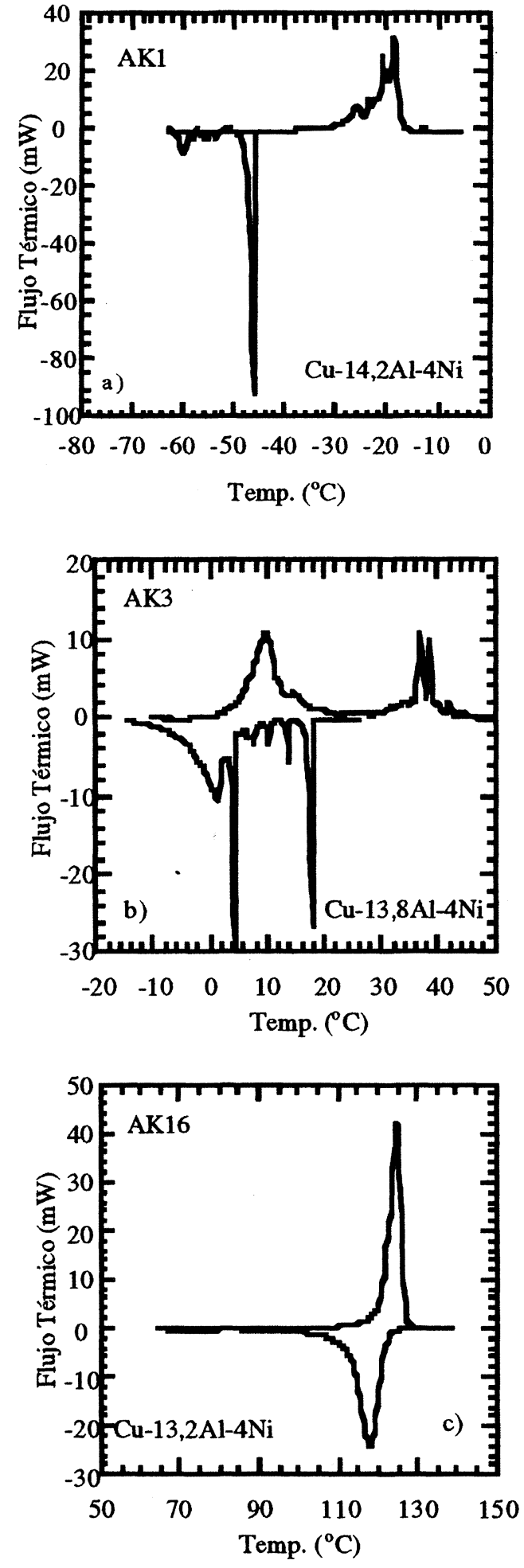

FIG. 1.- Termogramas D.S.C. de los tres diferentes tipos de transformaciones medidas.

a) Transformación $\left.\beta_{3} \Leftrightarrow \gamma^{6}{ }_{3} . b\right)$ Transformación $\beta_{3} \Leftrightarrow \beta^{\prime}{ }_{3}+\gamma^{6}{ }_{3} . c$ ) Transformación $\beta_{3} \Leftrightarrow \beta^{\prime}{ }_{3}$.

FIG. 1.- D.S.C. thermograms of the three different kinds of measured transformations .

a) Transformation $\beta_{3} \Leftrightarrow \gamma^{\prime}{ }^{3}$. b) Transformation $\beta_{3}$ $\Leftrightarrow \beta^{{ }^{\prime}}{ }_{3}+\gamma^{\prime}{ }_{3}$ c) Transformation $\beta_{3} \Leftrightarrow \beta^{\prime}{ }_{3}$.
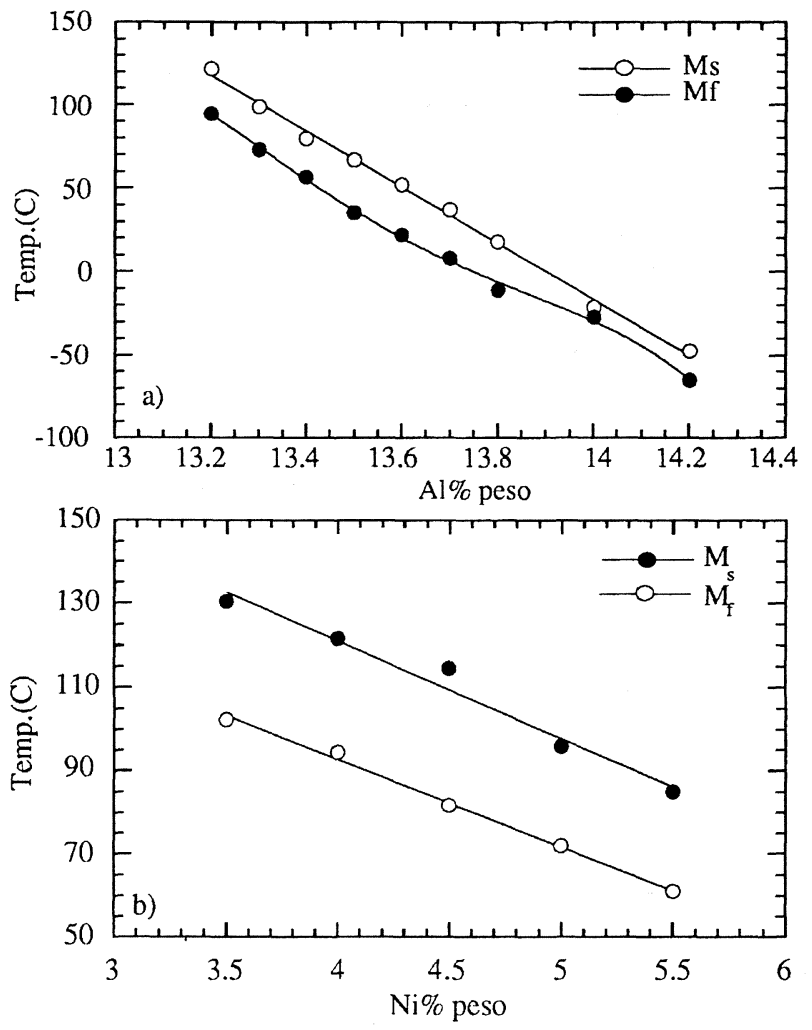

FIG. 2.- Temperaturas de transformación directa $M_{\mathrm{s}}$ y $\left.M_{\mathrm{f}} . a\right)$ Serie 1 y $b$ ) Serie 3.

FIG. 2.-Direct transformation temperatures $\mathbf{M}_{s}$ and $\mathrm{M}_{f}$ a) Serie 1 y b) Serie 3.

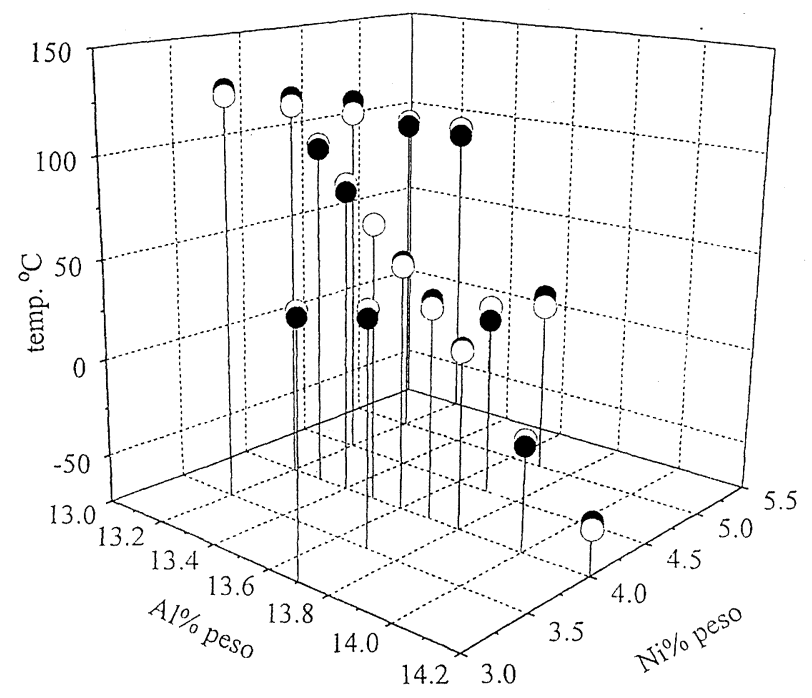

FIG. 3.- Temperaturas experimentales (puntos negros) y temperaturas obtenidas con la ec. [1] (puntos blancos) para las concentraciones utilizadas en este trabajo.

FIG. 3.- Measured (black points) and calculated (white points) temperatures using eq. [1] for the concentration range studied in this work. 
la extrapolación (Fig. 3). Como se puede ver, la temperatura depende acusadamente de la concentración, siendo ésta un factor determinante a la hora de precisar la temperatura de transformación. La dependencia de $M_{\mathrm{s}}$ con la concentración de aluminio, $170{ }^{\circ} \mathrm{C} / \%$, es casi diez veces mayor que la dependencia con el níquel. Además, dicha relación se ha comparado con más de 40 aleaciones dentro del rango de concentraciones de interés tecnológico ( 3,7 y 8$)$, entre el 13 y el $14,5 \%$ para el aluminio y el 2 y el $5 \%$ para el níquel, obteniéndose un buen acuerdo en todas ellas (5 y 9).

\section{CONCLUSIONES}

Mediante medidas de calorimetría D.S.C. se han determinado las temperaturas de transformación martensítica para una serie de monocristales de $\mathrm{Cu}$ Al-Ni. De esta forma, se ha obtenido una relación empírica entre la concentración de los elementos aleantes y las temperaturas de transformación martensítica aplicable dentro del rango de composiciones de interés tecnológico en las aleaciones de $\mathrm{Cu}$ Al-Ni. Se ha confirmado la concordancia de dicha relación empírica con los resultados obtenidos de la bibliografía.

\section{Agradecimiento}

Este trabajo se ha realizado gracias a la financiación de la CICYT en el marco del Plan Nacional de Materiales (Proyecto $n^{\circ}$ MAT-920353) y de la Universidad del País Vasco (Proyecto UPV 063.310EB049/95).

\section{REFERENCIAS}

(1) Pops, H. y Ridley, N. Metall. Trans. 1, 1970: 2.653

(2) Ahlers, M. Scr. Metall. 8, 213 (1974)

(3) Vasilenko, A.Yu., Sal'nikov, V.A. y Kosilov, A.T. Phys. Metals 4, 1982: 694.

(4) Ximing, Z., Xiong, L., Yun, Z., Jialong, M. y Yuantao, N. Proc. Int. Symp. Shape Memory Alloys, Gillin (China) 261 (1986)

(5) Recarte, V. Tesis Doctoral. Univ. del País Vasco. Bilbao (España) 1997.

(6) Ortin, J., Mañosa, L.L., Friend, C.M., Planes, A. y Yoshikawa, M. Phil. Mag. A 65, 1992: 461

(7) Friend, C.M., Ortin, J., Planes, A., Mañosa, Ll. y Yoshikawa, M. Scr. Metal. Mater. 24, 1990: 1641

(8) Sakamoto, H., Yoshikawa, M. y Shimizu, K. Mater. Trans. JIM 31, 1990: 848

(9) Recarte, V., Nó, M.L. y San Juan, J. Acta Mater. (Pendiente de publicación). 